\title{
Comparison of Gamma Radiation Crosslinking and Chemical Crosslinking on Properties of Methylcellulose Hydrogel
}

\author{
Sarawut Rimdusit ${ }^{1, *}$, Korapat Somsaeng ${ }^{1}$, Prartana Kewsuwan ${ }^{2}$, Chanchira Jubsilp $^{3}$, \\ and Sunan Tiptipakorn ${ }^{4}$ \\ 1 Polymer Engineering Laboratory, Department of Chemical Engineering, Faculty of Engineering, \\ Chulalongkorn University, Pathumwan, Bangkok 10330, Thailand \\ 2 Thailand Institute of Nuclear Technology (Public Organization), Saimoon, Ongkharak, Nakhonnayok \\ 26120, Thailand \\ 3 Department of Chemical Engineering, Faculty of Engineering, Srinakharinwirot University, \\ 107 Rangsit-Nakhonnayok Rd., Khong 16, Ongkharak, Nakhonnayok 26120, Thailand \\ 4 Department of Chemistry, Faculty of Liberal Arts and Science, Kasetsart University, Kamphaeng Saen, \\ Nakorn Pathom 73140, Thailand \\ E-mail: sarawut.r@chula.ac.th*
}

\begin{abstract}
In this research, characteristics of methylcellulose (MC) films modified with two crosslinking methods are investigated. The first method is the use of a glutaraldehyde (GA) crosslinker to promote hemi-acetal linkages between MC chains. The second one is gamma irradiation to form insoluble MC gel by intermolecular crosslinking. The effects of the MC concentration on the degree of crosslinking, water absorption, gel content, degree of swelling, and thermomechanical properties were determined. The results indicate that the chemically crosslinked MC films show lower polarity than the radiation crosslinked films. The nature of the crosslinkings was also revealed with FTIR spectra. The waterswelled films of chemically crosslinked MC were found to provide homogeneous gel structure whereas the radiation crosslinked MC films were observed to render less uniform crosslinked films.
\end{abstract}

Keywords: Gamma radiation, crosslinking, methylcellulose, hydrogel.

ENGINEERING JOURNAL Volume 16 Issue 4

Received 11 February 2012

Accepted 9 April 2012

Published 1 July 2012

Online at http://www.engj.org/

DOI:10.4186/ej.2012.16.4.15 


\section{Introduction}

In the past recent years, significant progress has been made on the development of biodegradable polymers for several key applications [1-5] such as packaging films, coatings, agriculture, and pharmaceutical materials as the production and the use of non-biodegradable plastics in the world have been enormously increased resulting in general shortage of landfill availability, and worsening the problems of the waste disposal. The increasing awareness for environmental conservation and protection has promoted the development of plastics that should degrade more rapidly in the environment, leading to a complete mineralization or bioassimilation of the plastics [5-7]. Biopolymers should be used in those applications where biodegradability and/or the derivation of natural resources gives added value, particularly, where valuable petroleum-based plastics are utilized with a short life time [8]. For these reasons, the biodegradable materials with controlled properties have been a subject of great research challenge to the community of material scientists and engineers [9-11].

Cellulose is the most abundant polysaccharide, and occurs mainly within the cell wall of higher plants as a structural material [12]. Cellulose is a linear macromolecule consisting of (1-4) glycosidic linkages of $\beta$ D-glucopyranosyl monomers, and this $\beta$-(1-4) configuration contributes to its rigid structure and to the formation of aggregates through intra- or inter-molecular hydrogen bonds via hydroxyl groups. Insolubility of cellulose in water is ascribed to this aggregation between the molecules, leading to the formation of highly ordered crystalline regions. Substitution of hydroxyl groups within the cellulose backbone by some functional groups provides cellulose with water-solubility through the reduction in the degree of crystallinity of the macromolecule. Representative cellulose derivatives include methylcellulose, carboxymethylcellulose, hydroxypropylcellulose, and hydroxy-propylmethylcellulose with many practical applications in industries, such as building, cosmetics, pharmaceuticals, and foods.

Methylcellulose (MC), a water-soluble carbohydrate polymer, is a modified type of cellulose with a propensity to form crosslinked three-dimensional network hydrogels that tend to swell in water or biological fluids. In recent years, MC is well known and of major interest to be used as environmental friendly products, especially as coatings or mulching films, because of its large availability, low cost, and easy processability. Moreover, MC has high solubility and efficient oxygen and lipid barrier properties. However, due to a highly biodegradable nature of MC, it can be used only in limited applications.

To modify the properties of MC film in order to broaden its useful properties, crosslinking is one of the most popular methods. Some polymer characteristics could be altered by crosslinking such as swelling degree, transport properties, mechanical properties, chemical stability, sponge structure as well as biodegradation rate [13-14]. There exist two major methods that can be used to generate crosslinking in water-soluble polymers. The first method is to promote covalent linkages between polymer chains by crosslinking agents such as glutaraldehyde (GA), a small molecule dialdehyde. The second method is by gamma irradiation of water-soluble polymer in aqueous solution, due to the high yield of radicals, to form hydrogel. Gamma irradiation, which is a very clean, easy and cost effective process, has been regarded as a very useful method in order to get macroscopic three dimensional, able to swell, lattice [14].

The main objective of this study is to compare the properties of crosslinked methylcellulose (MC) prepared by using glutaraldehyde $(\mathrm{GA})$ as a crosslinking agent and using gamma radiation. The effects of content of GA crosslinking agent and dose of gamma radiation on the obtained gel fraction, swelling degree, physical appearance of the gel, and thermomechanical properties of both crosslinked films will be examined.

\section{Experimental}

\subsection{Materials}

Methylcellulose (MC) powder, Tylose H 6000 YP2, was purchased from SE Tylose GmbH \& Co. KG., Germany. Glutaraldehyde $(\mathrm{GA})$ solution $\left(25 \mathrm{wt} \%\right.$, MW $=100.11$, BP $\left.=106^{\circ} \mathrm{C}\right)$ under trade name of UNILAB was obtained from Ajex Finechem, New Zealand. The degree of substitution (DS) of MC was in the range of 1.3 to 2.6 .

\subsection{Preparation of Chemically Crosslinked Methylcellulose Films}

Aqueous $\mathrm{MC}$ solution $(2 \mathrm{wt} \%)$ was prepared by dissolving $\mathrm{MC}$ powder in de-mineralized water and mixed for approximately 1 minute at $80^{\circ} \mathrm{C}$ using a homogenizer (IKA T25 basic) with a speed of 13,500 rpm. GA 
and hydrochloric acid were added after the MC solution was cooled to room temperature. The investigated GA contents were varied at $0.05,0.1,0.2,0.3$, and $0.5 \mathrm{wt} \%$. In each solution, 2 drops of hydrochloric acid were added to yield a solution of $\mathrm{pH} \sim 3$. The homogeneous blend solution was obtained using a magnetic stirrer at $700 \mathrm{rpm}$ for $1 \mathrm{hr}$. About $30 \mathrm{ml}$ of the obtained MC solution was poured into a polystyrene mold. Dried MC films were obtained after the solvent was evaporated in an air-circulated chamber at ambient temperature. Finally, the resulting dried films were washed by distilled water to neutralize the films. The thickness of each chemical crosslinked MC film was approximately $70 \mu \mathrm{m}$.

\subsection{Preparation of Radiation Crosslinked Methylcellulose Films}

Aqueous $\mathrm{MC}$ solution was prepared by dissolving $\mathrm{MC}$ powder in water and mixed about 1 minute at $80^{\circ} \mathrm{C}$ using a homogenizer (IKA T25 basic) with a speed of 13,500 rpm. About $20 \mathrm{ml}$ of the obtained MC solution was poured into a Petri dish. Gamma radiation experiment was performed at Thailand Institute of Nuclear Technology (Public Organization), Nakhonnayok province. Gamma ray was radiated on MC solutions at various doses of 5,10,15, 20 and $25 \mathrm{kGy}$. The MC dried films were obtained after the solvent was evaporated in an air-circulated chamber at ambient temperature. After evaporation, the films were sealed in polyethylene bags or poly(vinylidene chloride) packages for air-free irradiation, after degassing by a vacuum machine. The yielded thickness of the radiation crosslinked MC film was approximately $70 \mu \mathrm{m}$, same as that of the chemical crosslink films.

\subsection{Viscosity Measurement of Methylcellulose Solutions}

The viscosity at room temperature of MC solution (2-25 wt $\%$ ) before and after irradiation by gamma ray was measured by a rotational viscometer (Physica Rheolab MC1: Standard measuring system MS-Z3 $\mathrm{DIN} / \mathrm{MC1}$ ) at shear rates in the range of $10-1000 \mathrm{~s}^{-1}$ for $10 \mathrm{~min}$. The volume of the measured solution was about $20 \mathrm{ml}$.

\subsection{Moisture Absorption of Methylcellulose Films}

The moisture absorption testing based on ASTM D570-98 was performed to determine moisture absorption of the crosslinked MC films. The test samples with the dimension of $50 \mathrm{~mm} \times 50 \mathrm{~mm} \times 0.07 \mathrm{~mm}$ were dried at $60^{\circ} \mathrm{C}$ for $24 \mathrm{hr}$ and then were cooled down to room temperature in a desiccator, and immediately weighed as the initial weight. The samples were placed on the aluminum net inside a closed container at $100 \% \mathrm{RH}$ for $24 \mathrm{hr}$. After $24 \mathrm{hr}$, the samples were then weighed to determine the final weight. The moisture absorption of the sample was calculated according to Eq. (1):

$$
\text { Moisture absorption }(\%)=\frac{\left(\mathrm{W}_{\mathrm{f}}-\mathrm{W}_{\mathrm{i}}\right)}{\mathrm{W}_{\mathrm{i}}} \times 100
$$

where $\mathrm{W}_{\mathrm{i}}$ and $\mathrm{W}_{\mathrm{f}}$ are the weight of crosslinked MC films before and after absorbing moisture.

\subsection{Gel Fraction and Swelling Behaviour of Methylcellulose Samples}

Radiation and chemical crosslinked MC films were immersed and extracted in de-ionized water for about $72 \mathrm{hr}$ at room temperature, and then were dried at $60^{\circ} \mathrm{C}$ in a vacuum oven to constant weight. The gel fraction was calculated according to Eq. 2:

$$
\text { Gel fraction }(\%)=\left(\frac{G_{d}}{G_{i}}\right) \times 100
$$

where $G_{i}$ is the initial weight of dried sample, $G_{d}$ is the weight of dry gel after removing water. Radiation and chemically crosslinked MC hydrogels with certain weight were immersed into de-ionized water and taken out at certain time to measure the weight. Swelling degree was calculated as Eq. 3:

$$
\text { Swelling degree }=\frac{\left(G_{t}-G_{d}\right)}{G_{d}}
$$

where $G_{t}$ is the weight of the swollen hydrogel after swelled for 72 hours. 


\subsection{ATR FT-IR Spectroscopy of Methylcellulose Films}

To investigate functional groups and the structure of crosslinked MC films, FT-IR technique, NICOLET 6700 (Thermocorporation) equipped with CONTINUUM FT-IR microscope and a mercury cadmiumtelluride (MCT) detector, was used. The spectra were recorded using a resolution of $4 \mathrm{~cm}^{-1}$ and 128 scans. ATR spectra of crosslinked MC films were acquired using the diamond tip.

\subsection{Dynamic Mechanical Properties of Methylcellulose Samples}

Dynamic mechanical thermograms of crosslinked MC films were obtained by a dynamic mechanical analyzer from Netzsch Inc. (model DMA $242 \mathrm{C}$ ) under a tensile mode at a frequency of $1 \mathrm{~Hz}$. The samples were heated from 30 to $200^{\circ} \mathrm{C}$ at a rate of $2^{\circ} \mathrm{C} / \mathrm{min}$ in nitrogen atmosphere. The films with a dimension of $5 \mathrm{~mm} \times 10 \mathrm{~mm} \times 0.07 \mathrm{~mm}$ were subjected to sinusoidal deformation with $5 \mu \mathrm{m}$ amplitude. The glass transition temperature $\left(\mathrm{T}_{\mathrm{g}}\right)$ was taken as the maximum point on the loss tangent curve in the temperature sweep test.

\subsection{Thermal Degradation of Crosslinked Methylcellulose Films}

Degradation temperature $\left(T_{d}\right)$ and char yield of crosslinked MC films were investigated using a thermogravimetric analyzer (TGA) from Mettler-Toledo (Thailand) Co., Ltd. (model TGA/SDTA851e). The initial mass of each tested sample was $15-20 \mathrm{mg}$. The first heating scan, which was conducted to eliminate water residual, was carried out at a rate of $20^{\circ} \mathrm{C} / \mathrm{min}$ from room temperature to $120^{\circ} \mathrm{C}$. The second scan was heated from 40 to $800^{\circ} \mathrm{C}$ at a heating rate of $20^{\circ} \mathrm{C} /$ min under nitrogen atmosphere. The nitrogen purging flow rate was $100 \mathrm{ml} / \mathrm{min}$. Weight loss of a sample was measured as a function of temperature. The degradation temperature at $5 \%$ weight loss and the char yield at $800^{\circ} \mathrm{C}$ were recorded.

\section{Results and Discussion}

\subsection{Effect of Concentration of MC Solution on Radiation Crosslinking}

Irradiation of polysaccharide material evokes some effects depending on the type of polymers, parameters of irradiation, the phase of material under processing and others. The effects of ionizing radiation on polymeric materials can be manifested in one of three major ways. The polymer may undergo one or both of the two possible reactions: those that are molecular weight increasing in nature, or molecular weight reducing in nature. Or, in the case of radiation resistant polymers, no significant change in molecular weight will be observed. The conventional terms, which influence the final properties of polymers include: (a) scission of main chain, leading to diminishing of the molecular weight of macromolecules or degradation and (b) crosslinking, the opposite process to degradation, which leads to the formation of macroscopic, insoluble material [14-16].

To study the suitable concentration of MC solution that could undergo crosslinking reaction using gamma ray irradiation technique, Table 1 shows the gel formation and the rheological characteristics of the MC solution before and after gamma ray irradiation at the radiation dose of $5 \mathrm{kGy}$ at various concentrations of the MC solution. The numbers of MC concentration at low $\mathrm{wt} \%$ were closed to each other in order to determine the minimum concentration that could make the paste-like state to facilitate the film casting process. From the table, the viscosity of irradiated MC solution was greater than that of the un-irradiated solution when the concentration of MC solution was $4 \mathrm{wt} \%$ or higher. Moreover, for the concentration of $7 \mathrm{wt} \%$ or higher, gelation of the MC solution was also evidently observed. However, at lower concentration of $\mathrm{MC}$ solution i.e. at $2 \mathrm{wt} \%$, the opposite trend or the reduction in viscosity of the MC solution upon irradiation was observed. This phenomenon suggested that the crosslinking in the MC solution could be generated by gamma ray irradiation but at a solution concentration that was high enough i.e. at least $4 \mathrm{wt} \%$ in our case.

It is known that cellulose derivatives exposed to high energy irradiation either in dilute aqueous solution or in solid state predominantly lead to degradation of the polymer [17]. However, crosslinking process had been reported to be predominant under irradiation when a relatively concentrated solution, the so-called paste-like condition, was used. For example, Nagasawa et al. reported the solution concentration 
of carboxymethyl starch that favored crosslinking process to be in the range of 20 to $50 \mathrm{wt} \%$ which was in the paste-like condition above [18]. In our case, though the $4 \mathrm{wt} \%$ and $5 \mathrm{wt} \%$ solution could undergo viscosity enhancement, likely from the more predominant crosslinking reaction than the degradation process, the infinite gel network was unable to form under this radiation treatment. The infinite gel network, however, was obtained when MC solutions of $7 \mathrm{wt} \%$ and $25 \mathrm{wt} \%$ were used. In order to obtain uniform film thickness, a relatively high concentration of $25 \mathrm{wt} \%$, a paste-like state, was used to prepare the MC films for the rest of the radiation study in our work.

Table 1. Effect of concentration of MC solutions on gel formation and rheological characteristics.

\begin{tabular}{cccc}
\hline $\begin{array}{c}\text { Concentration } \\
\text { of MC solution } \\
\text { (wt\% based on water) }\end{array}$ & $\begin{array}{c}\text { Gel formation } \\
\text { after irradiation }\end{array}$ & & \multicolumn{2}{c}{ Viscosity at shear rate $\mathbf{1 2 5 s}^{\mathbf{- 1}(\mathbf{c P})}$} \\
\cline { 3 - 4 } before irradiation & $\begin{array}{c}\text { After irradiation } \\
\text { at 5kGy }\end{array}$ \\
\hline 2 & No & 819 & Very low \\
4 & Yes & 1707 & 3,257 \\
5 & Yes & 1989 & 4,389 \\
7 & Yes & 6010 & Gelled \\
25 & Yes & Paste-like & Gelled \\
\hline
\end{tabular}

\subsection{Moisture Absorption of Crosslinked MC Films}

The effects of doses of gamma ray $(\mathrm{kGy})$ and glutaraldehyde $(\mathrm{GA})$ crosslinker contents (wt $\%$ based on the $\mathrm{MC}$ ) on moisture absorption capacity of crosslinked MC films are presented in Fig. 1. The crosslinked MC films by gamma radiation were obtained using the radiation doses from $5 \mathrm{kGy}$ to $25 \mathrm{kG}$ whereas the GAcrosslinked films were obtained with the GA content ranging from $0.05 \mathrm{wt} \%$ to $0.5 \mathrm{wt} \%$. From the figure, the moisture absorption decreased with increasing dose of gamma ray and GA contents. The result showed that the moisture absorption systematically decreased from 160\% of the uncrosslinked MC films to $110 \%$ of the radiation crosslinked MC films at $25 \mathrm{kGy}$ of radiation dose and $119 \%$ of the $0.5 \mathrm{wt} \%$ GA crosslinked MC films. Therefore, both crosslinking techniques provided MC films with reduced degree of moisture uptake possibly due to the lower polarity in the resulting films. In those samples with increasing irradiation doses or GA contents, the degree of crosslinking of $\mathrm{MC}$ was expected to increase while the number of polar hydroxyl groups, a possible crosslinking site, of MC tended to decrease [19]. In addition, increasing degree of crosslinking might also lead to the reduction of the hydrogen bonds between water and MC thus causing the lower moisture uptake of the crosslinked films. This phenomenon was also similar to that of hydroxylpropyl cellulose (HPC) crosslinked with glutaraldehyde systems [15] in which the moisture absorption of the HPC film was found to decrease with increasing degree of crosslinking.

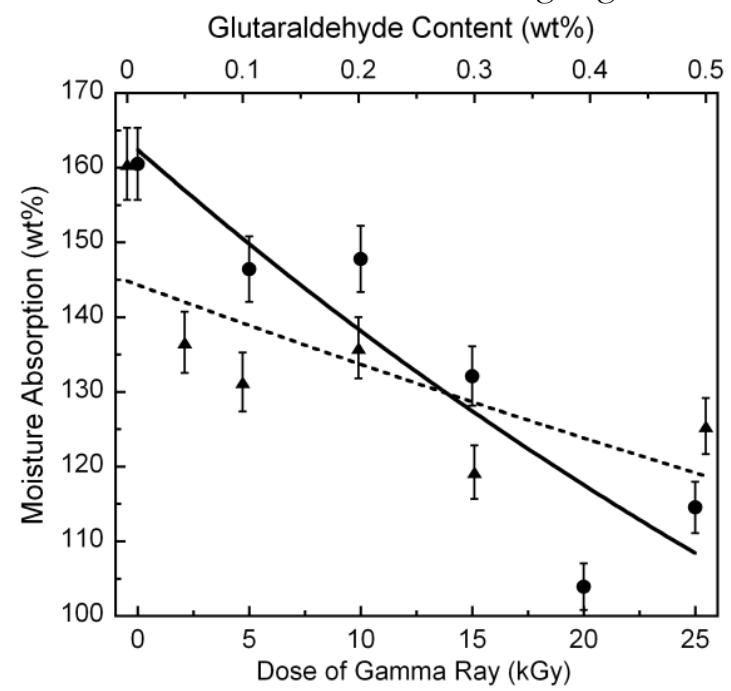

Fig. 1. Moisture absorption of crosslinked MC films at various doses of gamma ray and contents of glutaraldehyde $(\mathrm{GA}):(\bullet)$ radiation crosslinked films, $(\mathbf{\Lambda})$ chemically crosslinked films. 


\subsection{Gel Content of Crosslinked MC Films}

Gel content is defined as the amount of insoluble polymer in any solvent [14]. Gel contents of MC films at various doses of gamma ray ( $\mathrm{kGy}$ ) and those obtained at different contents of a GA crosslinker (\% by weight based on the MC) is presented in Fig. 2. In this figure, it was clearly seen that the gel contents of the crosslinked MC films increased with an increase of the doses of the gamma ray. The same trend was observed in the chemically crosslinked MC films with increasing the contents of a GA crosslinker. The gel content was determined to be $0 \%$ in the uncrosslinked MC sample due to its water soluble thermoplastic nature. The gel fraction; however, increased drastically from $0 \%$ to $80 \%$ using the radiation dose of only 5 $\mathrm{kGy}$ and the similar level of gel content i.e. $81 \%$ was obtained using a GA crosslinker at the content of only $0.05 \mathrm{wt} \%$. Both crosslinking techniques also revealed a slightly enhancement in gel content values with further crosslinking treatments. In the chemical crosslinking process, the gel content changed from $81 \%$ at the GA level of $0.05 \mathrm{wt} \%$ to the gel content value up to $90 \%$ at the maximum GA content of $0.5 \mathrm{wt} \%$. On the other hand, the gel content increased from $80 \%$ using a radiation dose of $5 \mathrm{kGy}$ to the value of $98 \%$ using a radiation dose of $40 \mathrm{kGy}$.

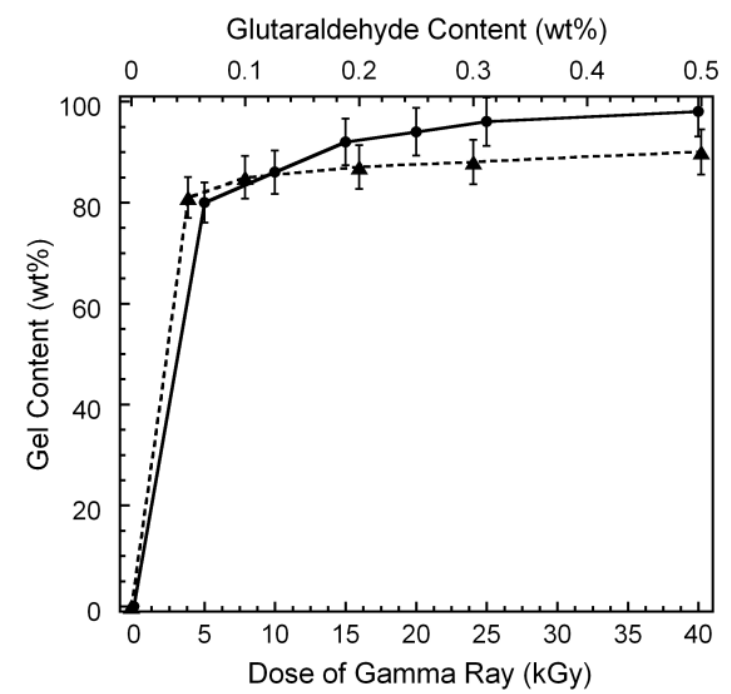

Fig. 2. Gel content of crosslinked MC gels at various doses of gamma ray and contents of glutaraldyhyde $(\mathrm{GA}):(\bullet)$ radiation crosslinked films, $(\boldsymbol{\Delta})$ chemically crosslinked films.

Gamma irradiation on cellulose derivatives typically lead to the random formation of free radicals on polymer chains and hydrogen atoms. These free radicals are responsible for such reactions as grafting and intermolecular crosslinking [16]. In addition, interactions of high-energy radiation with polysaccharides can result in both crosslinking and degradation by chain scission of the basic monomer units. An insoluble macroscopic gel is formed when crosslinking predominates chain-scission. In the case of GA crosslinked MC film, GA crosslinking occurs specifically at the hydroxyls group at $\mathrm{C}(2)$ or $\mathrm{C}(3)$ of anhydroglucose unit which should be in the neighbourhood of each other and can be linked by the GA molecule. Our results suggested that the adjacent hydroxyl groups had been mostly consumed at the $0.05 \mathrm{wt} \%$ of GA treatment to form an infinite network of MC films. Therefore, further addition of GA beyond this level did not show significant increase in their gel contents. However, greater level of gel content up to $98 \%$ can be obtained in the radiation crosslinking. However, within the experimental error, this may imply that the crosslinked sites from gamma irradiation can be generated at a slightly higher level than the crosslinked sites from GA treatment which is relatively fixed [15].

\subsection{Swelling Behaviours of Crosslinked MC Films}

Polymeric network which exhibits an ability to absorb and retain a significant amount of water within its structure but which does not dissolve is defined as hydrogel. Generally, a very important factor of the hydrogel for its advanced applications such as released control films, injectable polymers, and drug delivery 
system, is its swelling property. Swelling characteristics of polymers, usually presented as weight of solvent absorbed per $1 \mathrm{~g}$ of dried gel, strongly depends on hydrophilicity of the polymer, density of intermolecular links i.e. molecular weight of chain part between crosslinks and others.

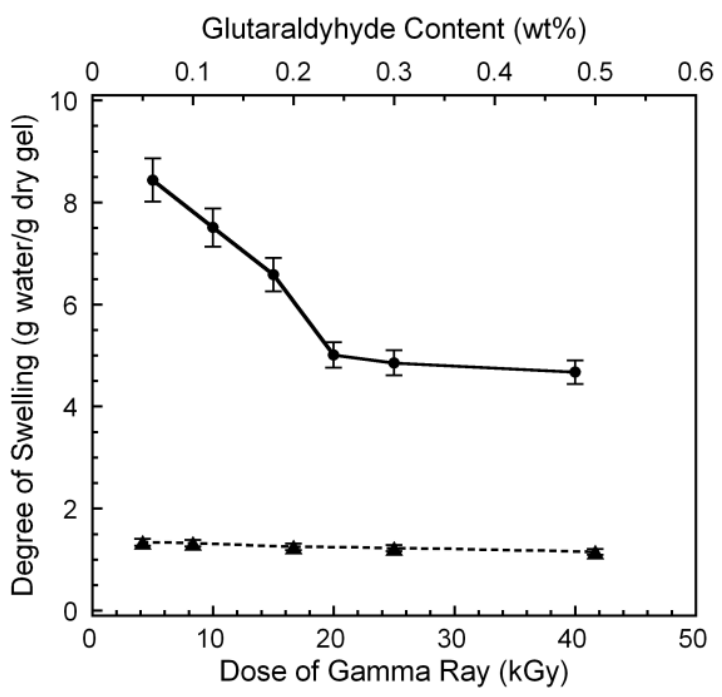

Fig. 3. Degree of swelling of crosslinked MC gels with different doses of gamma ray and content of glutaraldehyde: $(\bullet)$ radiation crosslinked gels, $(\mathbf{\Lambda})$ chemically crosslinked gels.

In this study, the relationship between the degree of swelling of MC film samples with doses of gamma ray as well as with contents of a GA crosslinker is illustrated in Fig. 3. From the figure, it was clearly seen that the degree of swelling of the crosslinked MC films decreased with increasing doses of gamma ray and the contents of a GA crosslinker. For irradiated MC films, using the radiation doses ranging from $5 \mathrm{kGy}$ to $20 \mathrm{kGy}$, the degree of swelling of the films decreased rapidly from $8.4 \mathrm{~g}$ water/g dry gel to $5.0 \mathrm{~g}$ water/g dry gel and then leveled off at $5.0 \mathrm{~g}$ water/g dry gel using radiation dose greater than $20 \mathrm{kGy}$. On the other hand, the GA crosslinked gels revealed a small dependence of degree of swelling on the GA contents. From the figure, the degree of swelling slightly decreased from $1.3 \mathrm{~g}$ water/g dry gel to $1.1 \mathrm{~g}$ water/g dry gel at contents of a GA crosslinker ranging from $0.05 \mathrm{wt} \%$ to $0.5 \mathrm{wt} \%$. Therefore, increasing the contents of GA crosslinker above $0.05 \mathrm{wt} \%$ showed negligible effect on degree of swelling of the resulting films. In comparison on the swelling values between radiation crosslinked and chemically crosslinked film samples, the swelling values of gamma irradiation crosslinked MC films were greater than that of GA crosslinked MC films. From these results, it is likely that the gamma irradiation crosslinking process should maintain more hydrophilic groups on the methylcellulose gel network than the GA crosslinking process thus resulting in the higher degree of swelling of the former process. This statement could be corresponded to the research of Florin et al. who studied about the effect of gamma radiation on the structure of MC and reported that the radiation induced chain cleavage, demethylation, carbonyl and acid group formation in MC, leading to enhanced hydrophilic behaviors. [20] Within the experimental error seen in Fig. 2, the gel content of MC after radiation was slightly higher than $\mathrm{MC}$ with chemical treatment. Therefore another factor that could make the degree of swelling of radiated MC higher than MC treated with GA could be the structure after radiation. In case of methyl cellulose after radiation, the chemical structure of methylcellulose has changed. As previously mentioned [20], it was reported that the radiation could induce acid group formation in MC. When the acid groups were formed, there has been an effect on the hydrophilic and swelling behaviors as observed in Figs. 2 and 3.

\subsection{FT-IR Spectroscopy of Crosslinked MC Films}

The FT-IR spectroscopic technique was used to specify crosslinking moieties in anhydroglucose unit and level of crosslinking in both crosslinking methods. The FT-IR spectra of pure MC film and radiation crosslinked MC films at various doses of gamma ray are showed in Fig. 4(a). Pure MC film had absorption bands related to $\mathrm{O}-\mathrm{H}$ stretching at $3447 \mathrm{~cm}^{-1},-\mathrm{CH}_{3}$ stretching on anhydroglucose unit at $2962 \mathrm{~cm}^{-1}$, 
$-\mathrm{CH}_{2}-$ stretching on anhydroglucose unit at $2860 \mathrm{~cm}^{-1}, \mathrm{C}-\mathrm{O}$ carbonyl stretching in the anhydroglucose unit of the cellulose at $1643 \mathrm{~cm}^{-1}, \mathrm{C}-\mathrm{OH}$ the in plane bend at $1440 \mathrm{~cm}^{-1}, \mathrm{CH}_{3}$ symmetric bend (umbrella mode) at $1375 \mathrm{~cm}^{-1}, \mathrm{C}-\mathrm{O}$ stretching from asymmetric oxygen bridge at $1163 \mathrm{~cm}^{-1}$, and ring stretching at $896 \mathrm{~cm}^{-1}$. These values were consistent with those reported by Wang et al. [21] and Rimdusit et al. [22]. In this figure, it can be observed that the peak at $3447 \mathrm{~cm}^{-1}\left(\mathrm{O}-\mathrm{H}\right.$ groups), $1643 \mathrm{~cm}^{-1}$ (C-O stretching in anhydroglucose unit), and $1440 \mathrm{~cm}^{-1}$ (C-OH groups in bend) decreased and the peak at $2860 \mathrm{~cm}^{-1}\left(-\mathrm{CH}_{2}-\right.$ stretching), and the peak at $1375\left(\mathrm{CH}_{3}\right.$ symmetric) increased with the increasing of doses of gamma ray. In this observation, it is likely that the free radicals were produced from dehydrogenation at $C(1), C(2), C(3)$, $\mathrm{C}(4), \mathrm{C}(5)$ and $\mathrm{C}(6)$ of anhydroglucose repeating units of $\mathrm{MC}$ chains that are the functional groups that could be dehydrogenated easier than the others [16]. Furthermore, the FT-IR spectrum of 40kGy of dose of gamma ray irradiated on MC film showed the decreasing on the peak at $2860 \mathrm{~cm}^{-1}\left(-\mathrm{CH}_{2}-\right.$ stretching) and $1163 \mathrm{~cm}^{-1}$ (C-O stretching from oxygen bridge) whereas the FT-IR spectrums of $15 \mathrm{kGy}$ and $25 \mathrm{kGy}$ of radiation crosslinked film showed negligible effect of height on peak at $1163 \mathrm{~cm}^{-1}(\mathrm{C}-\mathrm{O}$ stretching from glycosidic linkages). It implies that the high dose of gamma irradiation caused the degradation in polymer chains, breaking of chain inside the gel, which is directly reflected in degree of swelling. The same mechanism was found in MC and HEC crosslinked by gamma ray and electron beam irradiation reported by Wach et al.[14]. Evidently, swelling started to increase at higher doses, over 30kGy. It is known that after irradiation cellulose molecules are altered by breaking of glycosidic linkages and the introduction of carboxyl and carbonyl groups.
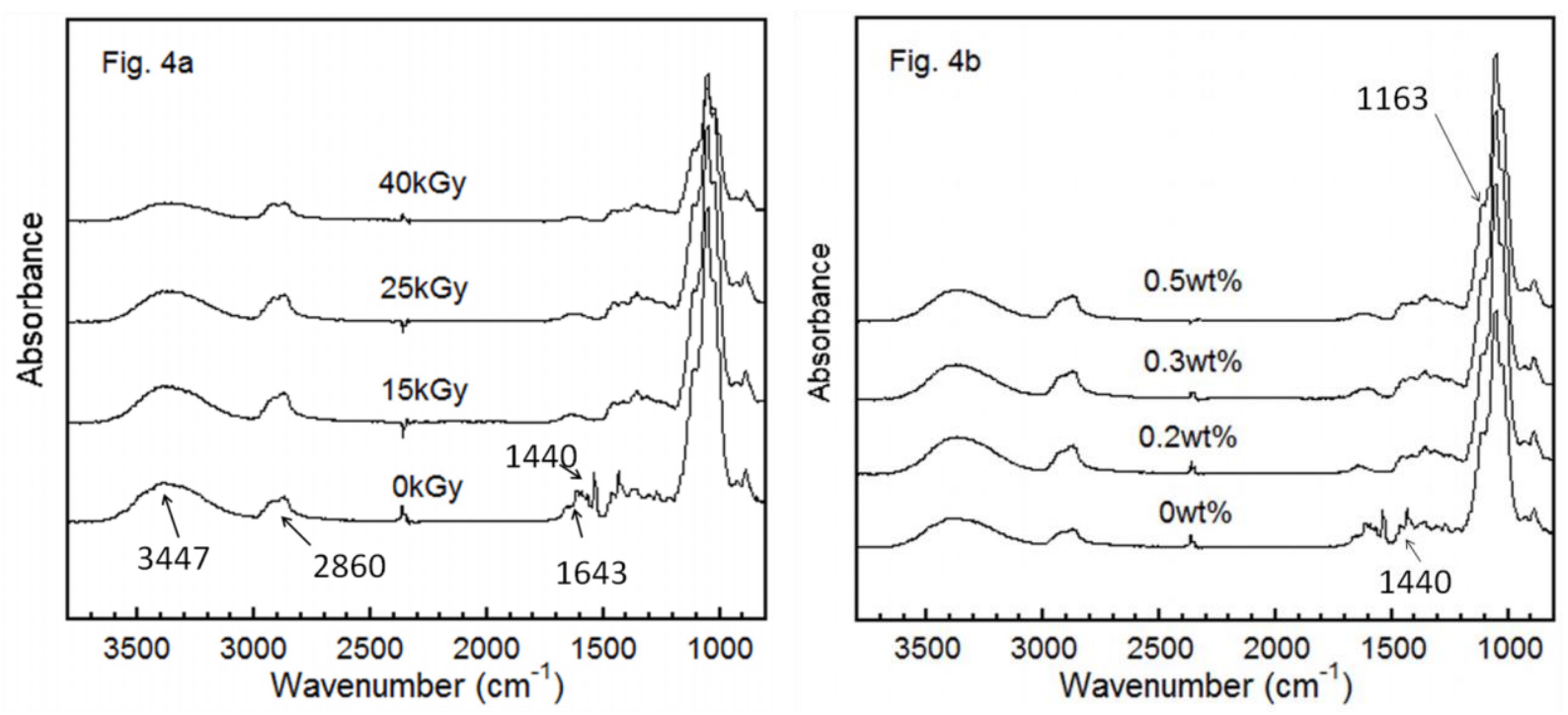

Fig.4. FT-IR spectra of crosslinked MC films: (a) pure MC film and radiation crosslinked MC films at various radiation doses, (b) pure MC film and chemically crosslinked MC films at different of glutaraldehyde contents.

The FT-IR spectra of pure MC film and chemically crosslinked MC films at various contents of a GA crosslinker are showed in Fig. 4(b). For chemically crosslinked MC films, the peak at $2860 \mathrm{~cm}^{-1}\left(-\mathrm{CH}_{2-}\right.$ stretching in alkane group of GA) and the peaks at $1710 \mathrm{~cm}^{-1}(\mathrm{C}=\mathrm{O}$ stretching from aldehyde group of GA) were used to indicate the crosslinking reaction. With an increase in the content of a GA crosslinker ranging from 0.2 to $0.5 \%$ by weight in the chemically crosslinked $\mathrm{MC}$, peak at $2860 \mathrm{~cm}^{-1}\left(-\mathrm{CH}_{2}-\right.$ from hemi-acetal crosslinked linkages) and the peak at $1163 \mathrm{~cm}^{-1}$ (C-O in hemi-acetal crosslinked linkages) slightly increased, but the peak at $1440 \mathrm{~cm}^{-1}$ (C-OH in plane bend) rapidly decreased. In this result, it can be explained that the dianldehyde groups of a GA crosslinker reacted with the hydroxyl groups at $C(2)$ and $C(3)$ in the anhydroglucose units of $\mathrm{MC}$ generating the three-dimensional crosslinked structure between MC chains. Moreover, the peak at $2860 \mathrm{~cm}^{-1}$ and the peak at $1163 \mathrm{~cm}^{-1}$ could imply the crosslink density of chemically crosslinked MC films that increased with the increasing of contents of a GA crosslinker. The appearance of the absorption spectrum also suggested the formation of intermolecular hydrogen bonding, resemble to the 
results reported for MC crosslinked with GA [22]. In addition, from the literature [20], the radiation induced acid group in MC; the FT-IR signal of COOH stretching could be observed at $1716 \mathrm{~cm}^{-1}$. It could be noticed that the height of the peak at this wave number was slightly increased with increasing radiation dose. That confirmed the effects of the radiation on the swelling results as previously observed.

\subsection{Visual Observation of Crosslinked MC Films}

The untreated MC film, chemically crosslinked MC film, and radiation crosslinked film are shown in Fig. 5(a), (c), and (e), respectively. From these figures, it can be seen that the chemically crosslinked MC film at $0.5 \mathrm{wt} \%$ of GA and the radiation crosslinked film at $25 \mathrm{kGy}$ of dose of gamma ray are clear transparent films. However, the surface textures of radiation MC films were rough. This phenomenon could possibly due to the fact as follows. For chemical crosslinking process, the solvent was gradually evaporated in an air-circulated chamber at ambient temperature, thus resulting in smooth films. For radiation-induced crosslinking process, the heat generated during the irradiation may result in initial evaporation of solvent which in turn leads to the increase of the viscosity of the MC solution, making it more difficult for the remaining solvent to evaporate later in air-circulated chamber at ambient temperature, hence resulting in samples with rough surfaces.

The water solubility of untreated MC film, chemically crosslinked MC film, and radiation crosslinked film is exhibited in Fig. 5(b), (d), and (f), respectively. These figures illustrate that pure MC film dissolved readily in water but both crosslinked MC films were insoluble in water. It was also observed that the volume of the swollen gel of the radiation crosslinked MC films was larger than that of the chemically crosslinked MC samples. This was maybe due to the radiation crosslinked hydrogel was more hydrophilic than the chemically crosslinked hydrogel. The appearances of radiation crosslinked hydrogel and chemically crosslinked hydrogel were also significantly different. The chemically crosslinked hydrogel rendered a relatively uniform gel possibly because of the homogeneous crosslinking in the gel to form a homogeneous network as shown in Fig. 5(d).

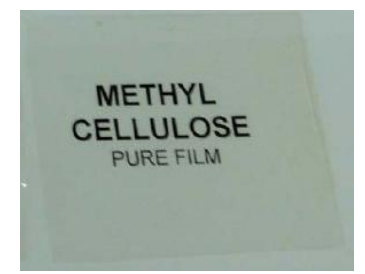

(a)

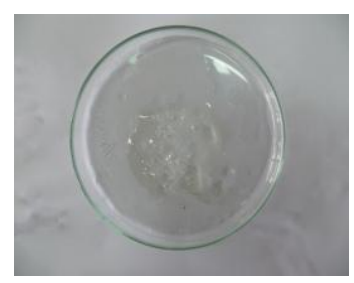

(b)

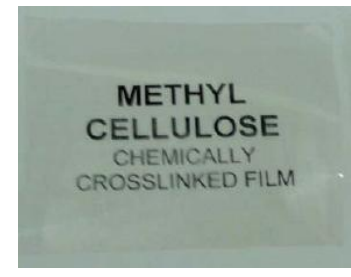

(c)

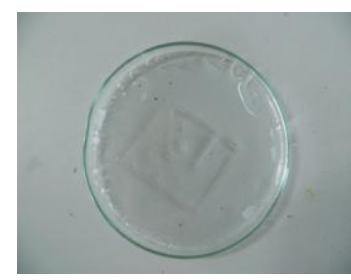

(d)

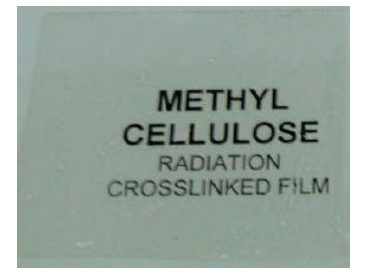

(e)

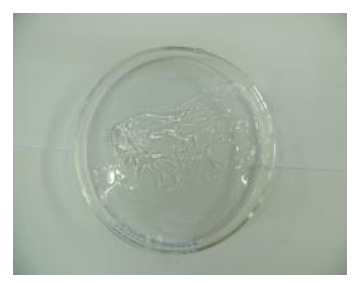

(f)

Fig. 5. Water solubility of methylcellulose films: (a, b) pure MC film, (c, d) chemically crosslinked MC film, (e, f) radiation crosslinked MC film.

The radiation crosslinked hydrogel; on the other hand, provided a rather non-uniform gel. The structure of the radiation crosslinked MC gel was likely a heterogeneous network which had crosslinked units locally concentrated in the hydrogel. Radiation crosslinked MC gel formed some gel clusters which were scattering in the MC gel sample as seen in Fig. 5(f). The reason for heterogeneous network formation in their gel samples was possibly due to the non-uniform radiation intensity irradiated on the specimen. The non-uniform radiation intensity might be caused by the irradiated condition of ${ }_{0} \mathrm{Co}$ gamma irradiator. The gamma ray which irradiated on stuck samples tended to scatter non-uniformly in the cell [23]. 


\subsection{Dynamic Mechanical Analysis of Crosslinked MC Films}

Dynamic mechanical analysis, which provides important Thermomechanical properties such as storage modulus $\left(\mathrm{E}^{\prime}\right)$, and loss tangent $(\tan \delta)$ of materials as a function of temperature, was employed to evaluate the effect of different compositions of GA contents and doses of gamma ray on the crosslinked MC films. Fig. 6 and Fig. 7 depict the variation of GA contents and doses of gamma ray had no significant effect on the storage modulus at room temperature $\left(30^{\circ} \mathrm{C}\right)$ of the obtained $\mathrm{MC}$ films maybe due to molecule structure of both crosslinked MC films complicated to inspect the real storage modulus of these films. In addition, at $200^{\circ} \mathrm{C}$ (temperature in the rubbery plateau region), the increasing of a GA crosslinker and dose of gamma ray tended to increase the plateau modulus of both crosslinked MC films. From the result, we can infer that GA addition and gamma irradiation can generate intermolecular crosslinking in the MC chains. An increase of storage modulus in the rubbery plateau region in principle relates to an increase in degree of crosslinking of the samples. The higher degree of crosslinking leads to denser network structures, which decreases the mobility of the polymer chains. Therefore, the higher crosslinked materials tend to have greater rigidity in nature. In addition, the effects of radiation as well as chemical treatments on the MC crosslinking are relatively indifferent.

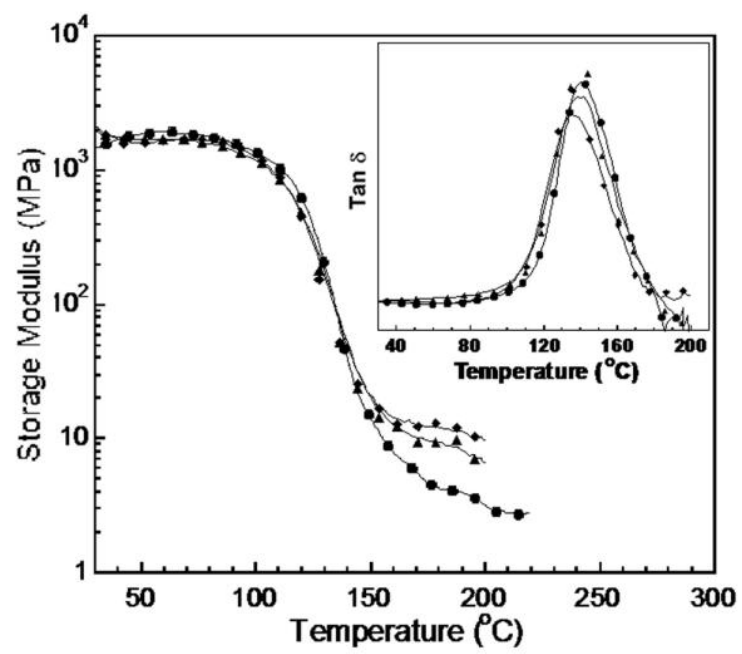

Fig. 6. Storage and loss tangent of chemically crosslinked MC films as a function of temperature at various contents of glutaraldehyde $(\mathrm{GA}):(\bullet) 0 \mathrm{wt} \%,(\boldsymbol{\bullet}) 0.1 \mathrm{wt} \%,(\mathbf{\Lambda}) 0.2 \mathrm{wt} \%$.

Inset in Fig. 6 and Fig. 7 illustrate the plots between the mechanical loss tangent $(\tan \delta)$ against temperature from the DMA experiment of the chemically crosslinked MC film and the radiation crosslinked MC films, respectively. The tan $\delta$ curves for the MC crosslinked films showed no effect on the $\alpha$-relaxation with increasing the contents of a GA crosslinker from $0.1 \mathrm{wt} \%$ to $0.2 \mathrm{wt} \%$ or with the doses of gamma ray from $5 \mathrm{kGy}$ to $25 \mathrm{kGy}$ i.e. the $\alpha$-relaxation peak corresponded to the glass transition temperature of the specimens and observed at $147^{\circ} \mathrm{C}$ for every specimen. This means that the glass transition temperature of the crosslinked $\mathrm{MC}$ films does not change with the levels of both crosslinking techniques used under this investigation. This phenomenon might also be attributed to the fact that the MC backbone was so rigid so that the network rigidity thus the glass transition temperature did not significantly change after crosslinking and the degree of the crosslinking may not be enhanced sufficiently to affect the glass transition temperature. Moreover, from the inset in Fig. 6 and Fig. 7, the heights of $\tan \delta$ are not different with the increase of doses of gamma ray and contents of a GA crosslinker. Generally, the $\tan \delta$ is related to the ratio between viscous and elastic behaviours. The reason that $\tan \delta$ is defined as the ratio of viscous components to elastic components, it can be assumed that the increased height is associated with the higher segmental mobility and more relaxing species as reported by Huang and Shi [24]. Therefore, it is possible to conclude that the variation of gamma irradiation in this investigation rendered negligible effects on the viscoelastic behaviour of the samples. While the contents of a GA crosslinker increased the height of $\tan \delta$ peak slightly decreased. It can be implied that the elastic behaviours in the chemically crosslinked films were higher than uncrosslinked films. 


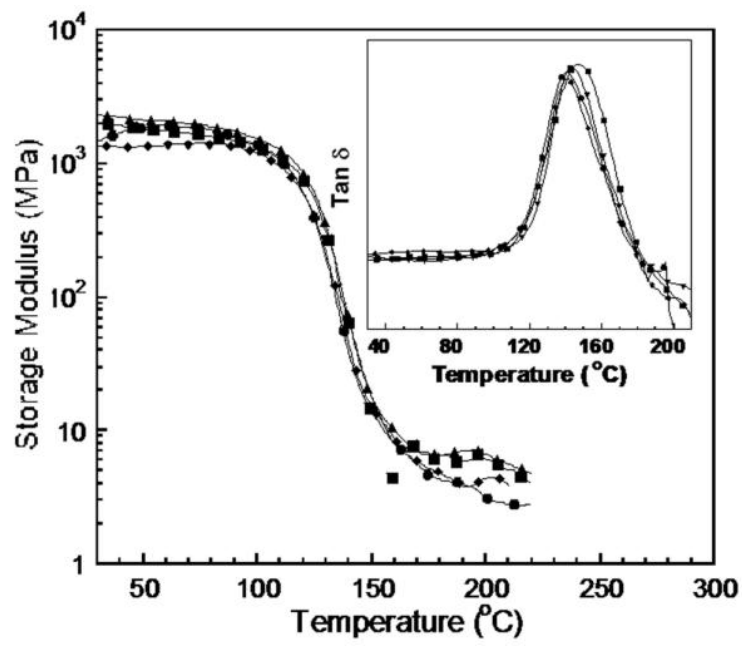

Fig. 7. Storage and loss tangent of radiation crosslinked MC films as a function of temperature at various doses of gamma ray: $(\bullet)$ k ky, (•) $5 \mathrm{kGy},(\bullet) 10 \mathrm{kGy},(\boldsymbol{\Delta}) 25 \mathrm{kGy}$.

\subsection{Termogravimetric analysis of crosslinked MC Films}

The TGA approach in which a sample loses mass with increasing temperature directly provided the information about the thermal stability and the degradation mechanism for crosslinked film. The TGA thermograms of the pure MC films, chemically crosslinked MC films at various contents of a GA crosslinker and radiation crosslinked MC films at various doses of gamma ray are presented in Fig. 8. and Fig. 9., respectively. It can be noticed that a slight weight loss $(\sim 3 \%-7 \mathrm{wt} \%)$ of $\mathrm{MC}$ and crosslinked MC started below $100^{\circ} \mathrm{C}$ which was reported that the possible causes for the initial weight loss are probably due to moisture and high water-retention capacity of MC [19]. In Fig.8, the contents of a GA crosslinker increased from $0.05 \mathrm{wt} \%$ to $0.5 \mathrm{wt} \%$ the degradation temperature (at $10 \%$ weight loss) of chemically crosslinked $\mathrm{MC}$ films increased from $310^{\circ} \mathrm{C}$ to $339^{\circ} \mathrm{C}$. While they show that the variation of doses of gamma ray had negligible effect on the thermal stability of crosslinked MC films as depicted in Fig. 9, respectively. From the result, it shows the effect of homogeneous crosslinked structures of chemically crosslinked MC films. The hemi-acetal linkages could slightly shift the thermal stability of MC films. However, the negligible effect of thermal stability on radiation crosslinked films might be caused of the heterogeneous crosslinked structure of films. From both figure, the major weight decrease of pure MC films and crosslinked MC films in both techniques took place in the temperature range of $310-410^{\circ} \mathrm{C}$ possibly because of the structure degradation of MC. In general, the thermal stability of a polymer is improved by crosslinking. However, in this case, thermal stability of MC gels remained comparable to that of the uncrosslinked MC because of the rigid anhydroglucose unit of MC. This phenomenon was similar to that reported by Park and Ruckenstein [19]. Beyond $450^{\circ} \mathrm{C}$, all curves were approaching a plateau value as mainly char residue remained. The residual weight of these samples at $700^{\circ} \mathrm{C}$ slightly increased with the increasing of doses of gamma ray and contents of a GA crosslinker. The increasing of char residues can be explained as due to the formation of the covalent bonding from intermolecular crosslinking of gamma irradiation and hemi-acetal formation of GA addition [24]. In addition, the further degradation of the cured film is considered to be a competition between the crosslinking of unsaturated species with radicals being formed (to form the char), and continued chain scission process occurring, evolving flammable materials which continue burning until there is nothing left. 


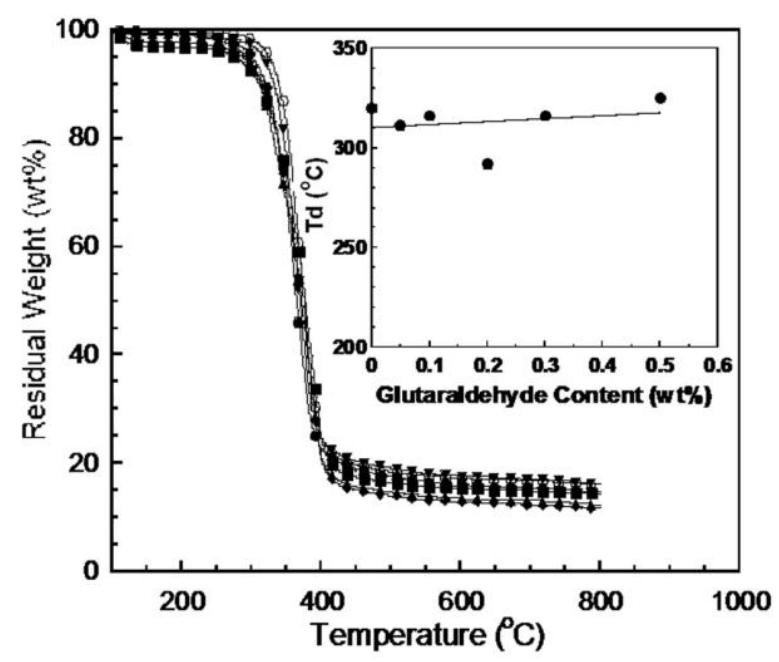

Fig. 8. TGA thermograms of chemically crosslinked MC films as a function of temperature at various contents of glutaraldehyde $(\mathrm{GA}):(\bullet) 0 \mathrm{wt} \%,(\boldsymbol{\bullet}) 0.05 \mathrm{wt} \%,(\bullet) 0.1 \mathrm{wt} \%,(\boldsymbol{\Delta}) 0.2 \mathrm{wt}^{0} \%,(\boldsymbol{\nabla}) 0.3 \mathrm{wt} \%$, (O) $0.5 \mathrm{wt} \%$.

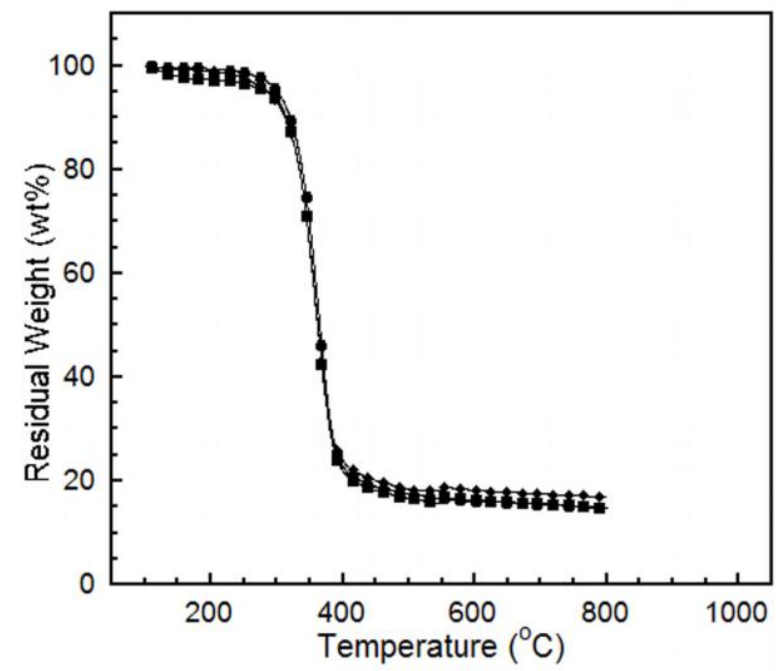

Fig. 9. TGA thermograms of radiation crosslinked MC films as a function of temperature at various doses of gamma ray: $(\bullet)$ kG $,(\bullet) 5 \mathrm{kGy},(\bullet) 10 \mathrm{kGy}$.

\section{Conclusions}

Crosslinked MC polymers prepared by gamma irradiation and GA addition were achieved. The results showed that the concentration of MC solution had significant effects on gel formation of radiation crosslinked MC hydrogels. The concentration of MC solution should be higher than $4 \%$ by weight of MC solution. The proper condition for radiation crosslinking to form $\mathrm{MC}$ hydrogel was $25 \mathrm{wt} \%$ of $\mathrm{MC}$ solution used in this study. Moisture absorption of radiation crosslinked film at various doses of gamma ray ranging from $0 \mathrm{kGy}$ to $25 \mathrm{kGy}$ decreased about $50 \%$ and chemically crosslinked film at various contents of GA crosslinker ranging from 0 to $0.5 \mathrm{wt} \%$ decreased about $40 \%$. In addition, the radiation crosslinking provided MC samples with greater gel content and degree of swelling than chemical crosslinking films. For radiation crosslinked MC films at $15 \mathrm{kGy}$ (at constant level of gel content), the gel contents rapidly enhanced to $92 \%$ but the gel contents of chemically crosslinked MC films at $0.05 \mathrm{wt} \%$ rapidly increased to $81 \%$. The degree of swelling of radiation crosslinked MC gel at $15 \mathrm{kGy}$ was $6.5 \mathrm{~g}$ water/g dry gel but the degree of swelling of chemically crosslinked MC gel at $0.05 \mathrm{wt} \%$ of GA was $1.3 \mathrm{~g}$ water/g dry gel. Furthermore, the FTIR spectra suggested that the radiation crosslinking had more chance to form crosslink networks and induced hydrophilicity than chemical crosslinking. In thermal properties, the various doses of gamma ray showed 
negligible effect on $T_{g}$ and $T_{d}$ of radiation crosslinked MC film while the GA contents in the range from $0.05 \mathrm{wt} \%$ to $0.5 \mathrm{wt} \%$ increased the decomposition temperatures of chemically crosslinked MC films increased from $310^{\circ} \mathrm{C}$ to $339^{\circ} \mathrm{C}$.

\section{Acknowledgements}

This research receives financial supports from the Research, Development and Engineering (RD\&E) Fund through National Nanotechnology Center (NANOTEC), National Science and Technology Development Agency (NSTDA), Thailand and the Higher Education Research Promotion and National Research University Project of Thailand, Office of the Higher Education Commission (AM1076A). The authors also greatly acknowledged the Thailand Institute of Nuclear Technology for the ${ }^{60} \mathrm{Co}$ gamma irradiation experiment.

\section{References}

[1] S. Fahs, M. Brogly, S. Bistac, and M. Schmitt, "Hydroxypropyl methylcellulose (HPMC) formaulated films: relevance to adhesion and friction surface properties," Carbohyd. Polym., vol. 80, pp. 105-114, 2010.

[2] A. P. Kumar and R. P. Singh, "Biocomposites of cellulose reinforced starch: Improvement of properties by photo-induced crosslinking," Bioresource Technol., vol. 99, pp. 8803-8809, 2008.

[3] P. Liu, J. Peng, J. Li, and J. Wu, "Radiation crosslinking of CMC-Na at low dose and its application as substitute for hydrogel," Radiat. Phys. Chem., vol. 72, pp. 635-638, 2005.

[4] A. P. Rokhade, N. B. Shelke, S. A. Patil, and T. M. Aminabhavi, "Novel interpenetrating polymer network microspheres of chitosan and methylcellulose for controlled release of theophylline," Carbohyd. Polym., vol. 69, pp. 678-687, 2007.

[5] R. Villalobos, P. Hernandez-Munoz, and A. Chiralt, "Effect of surfactants on water sorption and barrier properties of hydroxypropyl methylcellulose films," Food Hydrocol., vol. 20, pp. 502-509, 2006.

[6] R. Chandra and R. Rustgi, "Biodegradable polymers," Prog. Polym. Sci., vol. 23, pp 1273-1335, 1998.

[7] Q. Fang and M. A. Hanna, "Preparation and characterization of biodegradable copolyester-starch based foams," Bioresource Technol., vol. 78, pp. 115-122, 2001.

[8] M. Avella, J. J. Vlieger, M. E. Errico, S. Fischer, P. Vacca, and M. G. Volpe, "Biodegradable starch/clay nanocomposite films for food packaging application," Food Chem., vol. 93, pp. 467-474, 2005.

[9] B. Gupta and N. Revagade, "Poly(lactic acid) fiber: An overview," Prog. Polym. Sci., vol. 32, pp. 455482, 2007.

[10] S. I. Marras, I. Zuburtikudis, and C. Panayiotou, "Nanostructure vs. microstructure: morphological and thermomechanical characterization of poly(L-lactic acid)/layered silicate hybrids," Europ. Polym. J., vol. 43, pp. 2191-2206, 2007.

[11] L. Zhao, F. Luo, J. M. Wasikiewicz, H. Mitomo, N. Nagasawa, T. Yagi, M. Tamada, and F. Yoshii, "Adsorption of humic acid from aqueous solution onto irradiation-crosslinked carboxymethylchitosan," Bioresourec Technol., vol. 99, pp. 1911-1917, 2008.

[12] D. G. Coffey, D. A. Bell, and A. Henderson, "Cellulose and cellulose derivatives," in Food Polysaccharides and their Applications, A. M. Stephen, Ed. New York: Marcel Dekker, Inc., 1995, pp. 123153.

[13] V. Coma, I. Sebti, P. Pardon, F. H. Pichavant, and A. Deschamps, "Film properties from crosslinking of cellulosic derivatives with a polyfunctional carboxylic acid," Carbohyd. Polym., vol. 51, pp. 265-271, 2003.

[14] R. A. Wach, H. Mitomo, N. Nagasawa, and F. Yoshii, "Radiation crosslinking of methylcellulose and hydroxyethylcellulose in concentrated aqueous solutions," Nucl. Instr. Meth. Phys. Res. B, vol. 211, pp. 533-544, 2003.

[15] N. Pekel, F. Yoshii, T. Kume, and O. Guven, "Radiation crosslinking of biodegradable hydroxypropylmethylcellulose," Carbohyd. Polym., vol. 55, pp. 139-147, 2004.

[16] R. A. Wach, H. Mitomo, N. Nagasawa, and F. Yoshii, "Radiation crosslinking of carboxymethylcellulose of various degree of substitution at high concentration in aqueous solutions of natural pH," Radiat. Phys. Chem., vol. 68, pp. 771-779, 2003. 
[17] B. Fei, R. W. Wach, H. Mitomo, F. Yoshii, and T. Kume, "Hydrogel of biodegradable cellulose derivatives. I. Radiation- induced crosslinking of CMC," J. Appl. Polym. Sci., vol. 78, pp. 278-283, 2000.

[18] N. Nagasawa, T. Yagi, T. Kume, and F. Yoshii, "Radiation crosslinking of carboxymethy starch," Carbobydr. Polym., vol. 58, pp. 109-113, 2004.

[19] J. S. Park and E. Ruckenstein, "Viscoelastic properties of plasticized methylcellulose and chemically crosslinked methylcellulose," Carbohyd. Polym., vol. 46, pp. 373-381, 2001.

[20] F. A. Blouin, V. J. Ott, T. Mares, and J. C. Arthur, "The effects of gamma radiation on the chemical properties of methylcellulose," Text. Res. J., vol. 34, pp. 153-158, 1964.

[21] X. L. Wang, K. K. Yang, Y. Z. Wang, D. Y. Wang, and Z. Yang, "Crystallization and morphology of a novel biodegradable polymer system: Poly(1,4-dioxan-2-one)/starch blends," Acta. Materialia., vol. 52, pp. 4899-4905, 2004.

[22] S. Rimdusit, S. Jingjid, S. Damrongsakkul, S. Tiptipakorn, and T. Takeichi, "Biodegradability and property characterization of methyl cellulose: effect of nanocompositing and chemical crosslinking," Carbohyd. Polym., vol. 72, pp. 444-455, 2008.

[23] Y. Osada and K. Kajiwara, Gel Handbook. San Diego: Academic Press, pp. 4-20.

[24] Z. Huang and W. Shi, "UV curing behaviour of hyperbranched polyphosphate acrylate/di(hydroxypropyl methacrylate) piperazine and properties of the cured film," Prog. Org. Coat., vol. 59, pp. 312-317, 2007. 